\title{
Real time estimation of chlorophyll content based on vegetation indices derived from multispectral UAV in the kinnow orchard
}

\author{
Muhammad Naveed Tahir ${ }^{1 *}$, Syed Zaigham Abbas Naqvi ${ }^{1}$, Yubin Lan ${ }^{2,3,4}$, Yali Zhang ${ }^{2}$, \\ Yingkuan Wang ${ }^{5}$, Muhammad Afzal ${ }^{6}$, Muhammad Jehanzeb Masud Cheema ${ }^{7}$, Shahid Amir ${ }^{8}$ \\ (1. Department of Agronomy, PMAS-Arid Agriculture University, Rawalpindi, 46300, Pakistan; \\ 2. National Center for International Collaboration Research on Precision Agricultural Aviation Pesticides Spraying Technology/ \\ College of Engineering, South China Agricultural University, Guangzhou 510642, China; \\ 3. Department of Biological and Agricultural Engineering, Texas A\&M University, College Station, Texas, 77843, USA; \\ 4. Texas A\&M AgriLife Research and Extension Center, Beaumont, Texas, 77713, USA; \\ 5. Chinese Academy of Agricultural Engineering Planning and Design, Beijing 100125, China; \\ 6. Department of Geography and Environmental Science, the University of Reading, Whiteknights, PO Box 227, Reading, \\ RG6 6AB, United Kingdom; \\ 7. U.S. Pakistan Center for Advanced Studies in Agricultural Food Security, University of Agriculture, Faisalabad, Pakistan; \\ 8. Institute of Geo-informatics and earth observation, PMAS-Arid Agriculture University, Rawalpindi, 46300, Pakistan)
}

\begin{abstract}
Nondestructive estimation of the biophysical properties of crops provide quick and real time information of crop health under wide range of environment. The chlorophyll content is an important indicator of crop health and widely used for determination of nutritional status of the crops real time in precision agriculture. Advancement in the low altitude remote sensing (LARS) technologies such as Unmanned Aerial vehicles (UAVs) provides high temporal and spatial resolution solution for nondestructive, rapid and accurate estimation of biophysical properties of various crops. The main objective of this study was to evaluate the high resolution multispectral UAV images for nondestructive and real time estimation of the kinnow tree leaves chlorophyll content in district Sargodha, Pakistan. Kinnow tree leaves chlorophyll contents were measured manually using chlorophyll meter (SPAD-502 Minolta) in the kinnow orchard along with GPS positions in district Sargodha. The UAVs images were also acquired during the same time when ground-truthing campaign for kinnow leaves chlorophyll content was performed. Vegetation indices including Normalized Difference Vegetation Index (NDVI), Transformed Normalized Difference Vegetation Index (TNDVI), Modified Chlorophyll Absorbed Ratio Index (MCARI2), Soil adjusted vegetation Index (SAVI) and Modified soil adjusted vegetation index (MSAVI2) were derived by multispectral UAV images for chlorophyll estimation. The regression analysis was performed between ground-truthing data of chlorophyll content and UAV derived vegetation indices for predicting kinnow leave chlorophyll content model. MSAVI2 and TNDVI were proved to be more robust indices to estimate the chlorophyll content in the kinnow orchard with the highest coefficients of determination $\left(R^{2}\right) 0.89$ and 0.85 respectively. The results showed that the multispectral UAV can be used for accurately estimation of chlorophyll content and assess crop health status in a wider range which will help in managing crop nutrition requirement in real time in the kinnow orchard.
\end{abstract}

Keywords: Chlorophyll content, kinnow orchard, Multispectral UAV, Vegetation indices DOI: $10.33440 /$ j.ijpaa.20180101.0001

Citation: Tahir M N, Naqvi S Z A, Lan Y B, Zhang Y L, Wang Y K, Afzal M, et al. Real time monitoring chlorophyll content based on vegetation indices derived from multispectral UAVs in the kinnow orchard. Int J Precis Agric Aviat, 2018; 1(1): 24-31.

\section{Introduction}

contents can help indirect nitrogen status of crop (Moran et al.,

In plants, chlorophyll is the most important pigment for photosynthesis (Yuan et al., 2007). Chlorophyll converts solar energy into chemical energy, so it was reported that chlorophyll contents are directly correlated with crop growth and yield. Few studies showed that leaf nitrogen content is positively correlated with chlorophyll content. Therefore, estimation of chlorophyll

Received date: 2018-03-15 Accepted date: 2018-06-18

Biographies: Syed Zaigham Abbas Naqvi, Postgraduate student, research interests: remote sensing, Email: zaigham572@mail.com; Yubin Lan, PhD, professor, research interests: precision agricultural aviation application, Email: ylan@scau.edu.cn; Yali Zhang, PhD, Associate Professor, research Interests: instrumentation and control, agricultural smart sensors, Email: ylzhang@ scau.edu.cn; Yingkuan Wang, PhD, Research Professor, research interests: agricultural mechanization, automation and information, Email: wykford@ 188.com; Muhammad Afzal, PhD, Associate Professor, research interests: 
2000).

Conventional way for pigmentation analysis including spectrophotometer, destruction of leaves or high performance liquid chromatography (HPLC), and therefore cannot measure changes in pigmentation of individual leaves over time. In addition, these technologies are time-consuming and expensive, so it is impractical to assess the health status of the crops. Therefore, accurate, efficient, and practical methods are needed to estimate this biophysical parameter.

The use of Precision Agriculture (PA) technologies is considered one of the key components in modern agricultural development for improving the crop production at farm level. Some of the perceived benefits of PA include increasing crop yield and efficiency by lowering the costs associated with fertilizer, pesticides, herbicides, and fungicides. An additional socio-economic benefit of PA is reducing the transport of agriculture inputs on the air, soil and water.

A variety of highly resolution satellite data (IKONOS, QuickBird, GeoEye-1 and WorldView-2) [6-16] is available but their satellites' poor temporal resolution still a barrier to fully utilized this system efficiently. In addition, the costs and availability of high resolution satellite imagery often limit their applications in PA (Wu et al. 2007). Consequently, Unmanned Aerial Vehicles (UAVs), which are more manoeuvrable, cheaper to operate, and require less capital costs, may serve to address this need. Unmanned Aerial System (UAS) could be an inexpensive and more practical substitute for satellite and general aviation aircraft for high resolution remotely sensed data. Moreover, UAS are immediately accessible as a tool for remote sensing scientists and farmers (Swain et al. 2010). In recent years, small commercial UAS (<50 kg) (Laliberte and Rango 2011) have been available for environmental and agricultural applications. Furthermore, the rapid development of Low Altitude Remote Sensing Systems (LARS) over the past decade makes its application for PA possible.

A wide variety of UAVs are, using extensively in military and civilian applications (Blyenburgh, 1999). Applications include archaeological prospecting (Eisenbeiss, 2004), rangeland management (Hardin and Jackson, 2005), assessment of grain crop attributes (Jensen et al., 2003; Hunt et al., 2005), and vineyard management (Johnson et al., 2001). In agriculture, UAVs have been used for pest control and remote sensing (Huang et al. 2009). Moreover, UAS are immediately accessible as a tool for remote sensing scientists and farmers (Swain et al. 2010). These system still did not completely meet the requirement for real time monitoring crop health status due to clouds, aerosols, water vaporous and most important spatial and temporal resolution still a barrier to fully utilized this system efficiently.

Leaf-reflection based non-destructive techniques, a robust and simple method have been proposed as an alternative to pigment quantification in leaves (Richardson et al., 2002; Sims \& Gamon, 2002; Gitelson et al., 2003; Hu et al., 2004; Le Maire et al., 2004)

simulation modeling and remote sensing, Email: drmuhammadafzal100 @gmail.com; Muhammad Jehanzeb Masud Cheema, PhD, Agricultural Engineer, research interest: precision agriculture, Email: mjm.cheema@ uaf.edu.pk; Shahid Amir, PhD scholar, research interest: remote sensing and GIS, Email: spacian718@gmail.com.

*Corresponding author: Muhammad Naveed Tahir, $\mathrm{PhD}$, Assistant Professor, Research Interest: Remote sensing and Precision Agriculture. Department of Agronomy, PMAS-Arid Agriculture University. Rawalpindi, 46300, Pakistan. Email: naveed@uaar.edu.pk. and in canopies (Barton, 2001; Gitelson et al., 2005). Efforts has been made to develop relationship between leaf chlorophyll and plant reflectance (Tahir et al. 2013). But to represent chlorophyll content at canopy level are still uncertainties. There is need to accurate, rapid, and practical methods to estimate chlorophyll content per unit ground canopy in the kinnow orchards. The main objectives of this study was to real time estimation of the kinnow tree leaves chlorophyll content base on vegetation indices derived from multispectral UAVs and compared with the ground-truthing chlorophyll contents measured using SPAD chlorophyll meter for developing prediction model of leaf chlorophyll contents in real time.

\section{Material and methods}

\subsection{Study area and ground-truthing data of chlorophyll content measurement}

The current study was conducted at Kotmomin, district Sargodha, having latitude $32^{\circ} 01^{\prime} 00^{\prime \prime}$ North and longitude $73^{\circ} 02^{\prime} 30^{\prime \prime}$ East (Figure 1). Being a largest producer of the kinnow, it is $11^{\text {th }}$ biggest city of Pakistan and $6^{\text {th }}$ of Punjab, occupying $5,864 \mathrm{~km}^{2}$ area and 2,665,979 population of which only $28 \%$ lived in urban area according to 1998 Pakistan census. In summer the temperature rises up to $50{ }^{\circ} \mathrm{C}$ while in winter it drops below the freezing point. Most common crops cultivated in the district are the kinnow, wheat, rice, and sugarcane which are exported nationally and internationally. 

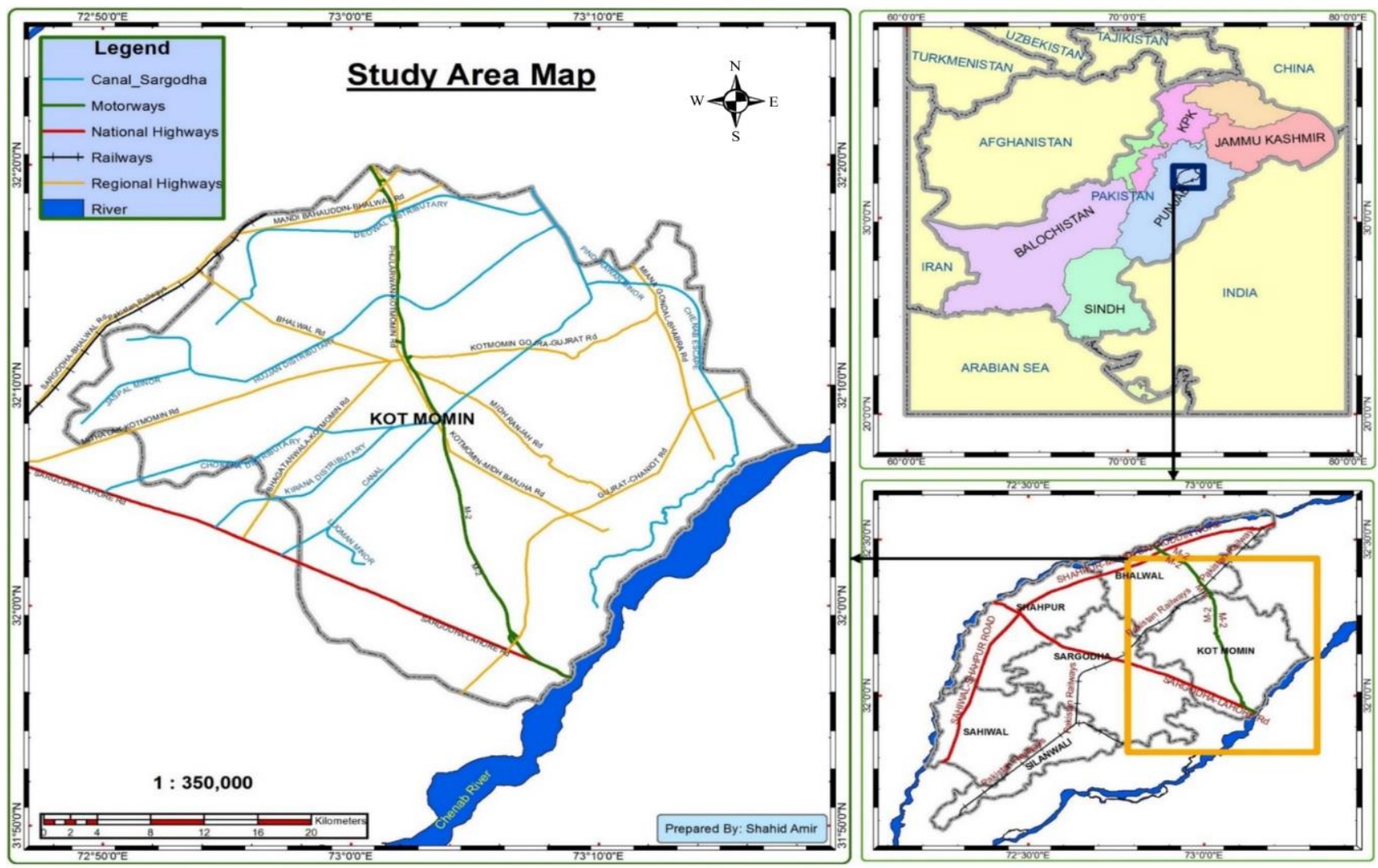

Figure 1 Study location map

Generally, the citrus orchards in Pakistan contain 100 trees per acre, separated by 6 meter from each other with average height of 4-5 meters and diameter of 3-7 meters. Ideally during the season (yearly) citrus field is irrigated 4-5 times and fertilized 2 times. The Kotmomin has been blessed a variety of soils from sandy to clay, hence there are some micro climatic zones.

The field visit was performed in April 26, 2018 to collect the kinnow tree leaves chlorophyll content data by using chlorophyll meter (SPAD-502 Minolta) for in situ measurement of the kinnow leaves chlorophyll contents from 46 trees randomly (Figure 2). Seven different leaves at different points on one kinnow plant were selected for chlorophyll content measurement and then took the averaged value of them. The sample locations were geo-located by using GPS meter. General information like tree height, age, average yield, previous status, and nutrient applications has also been obtained by interviewing by the farmers.

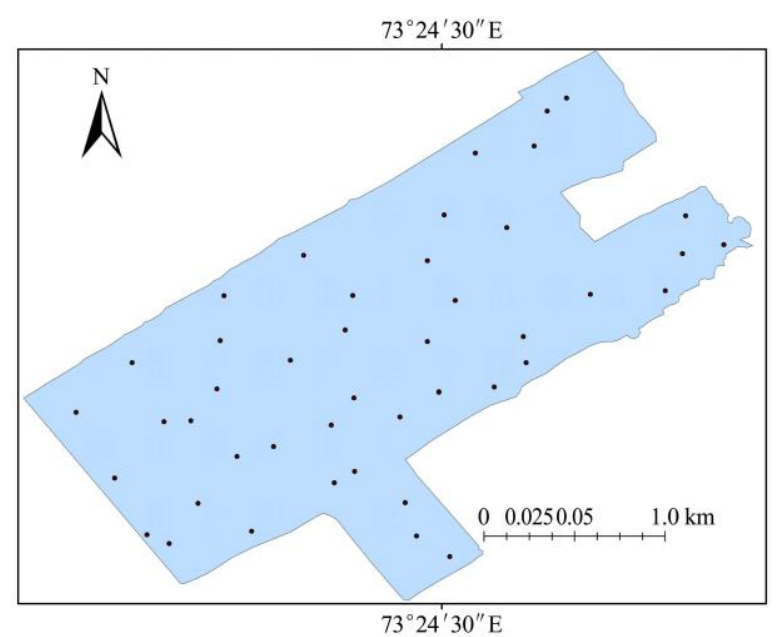

Figure 2 Point data map of chlorophyll content measurement by SPAD-502 chlorophyll meter at Kotmomim in the kinnow orchard

\subsection{UAVs system and flight data acquiring}

The eBee Agdesigned as a fixed wing UAVs for application in precision agriculture has a payload of $150 \mathrm{~g}$. This UAVs was equipped with MultiSpec 4C camera developed by Airinov (Airinov, 75018 Paris, France, www.airinov.fr/en/uav-sensor/ agrosensor/) and customized for the eBee Ag. It contains four distinct bands with no spectral overlap $(530-810 \mathrm{~nm})$ : green, red, red-edge, and near infrared bands, and is controlled by the eBee Ag autopilot during the flight (Table 1). The eBee MultiSpec 4C camera had a predefined setting by Sensefly; ISO and shutter speed was set to automatic, maximum aperture was set to $f / 1.8$ and focal distance was fixed at $4 \mathrm{~mm}$.

Table 1 eBee Ag Sensor Specification

\begin{tabular}{ccccll}
\hline Sensor & $\begin{array}{c}\text { Platform } \\
\text { (UAVs) }\end{array}$ & $\begin{array}{c}\text { Sensor } \\
\text { resolution } \\
\text { /MP }\end{array}$ & $\begin{array}{c}\text { Focal } \\
\text { length } \\
\text { /mm }\end{array}$ & $\begin{array}{c}\text { Full width at half } \\
\text { maximum (FWHM) }\end{array}$ & $\begin{array}{c}\text { Peak } \\
\text { wavelength }\end{array}$ \\
\hline MultiSpec & eBee Ag & $\begin{array}{c}1.2 \text { (four } \\
\text { sensors) }\end{array}$ & 3.6 & $\begin{array}{l}\text { Green: 530-570 } \\
\text { Red: 640-680 }\end{array}$ & $\begin{array}{l}\text { Green: } 550 \\
\text { Red: 660 }\end{array}$ \\
4C & & & & NIR: 770-810 & NIR: 790 \\
\end{tabular}

\subsection{Reflectance calibration panel}

For radiometric calibration, spectra of easily recognizable objects (e.g. gray scale calibration board) are needed. A black-gray-white grayscale board with known reflectance values was built and placed in the field during flights for further image calibration. This grayscale calibration panel met the requirements for further radiometric calibration including (1) the panel was spectrally homogenous, (2) it was near Lambertian and horizontal, (3) it covered an area many times larger than the pixel size of the Canon S100, and (4) covered a range of reflectance values [25].

The flights were carried out at kotmomin in district Sargodha on April 26, 2018 in the kinnow orchard with eBee UAS (senseFly, Switzerland). All flights were carried out in stable ambient light 
conditions from 12:00 pm to 2:00 pm, with excellent visibility and a wind below $5 \mathrm{~m} / \mathrm{s}$, at flight altitude of $41 \mathrm{~m}$ (above ground level). The imaged area of the kinnow field, including the surroundings, is about 11 hactare. The time needed for a single flight of the UAS imaging was 12 minutes. At the time of images, two flights were carried out, the first one by a Canon Powershot S110 photo camera (visible spectrum, RGB-red/green/blue) for visible RGB image (orthophoto) to run a rapid analysis for visual orchard variability. A second fly with a Canon Powershot S110 NIR camera (near infra-red [NIR], near infra-red/green/blue) that provides the maximum absorption peaks at $550 \mathrm{~nm}$ (green), $625 \mathrm{~nm}$ (red) and $850 \mathrm{~nm}$ (NIR) wavelengths respectively, allowing the computation of Vis both in the visible and near infrared. The technical features of the S110 RGB or the S110 NIR involve resolution of 12 million pixels, a weight of $0.7 \mathrm{~kg}$, sensor size of $7.44 \times 5.58 \mathrm{~mm}^{2}$, pixel pitch of $1.33 \mu \mathrm{m}$ and image format in RAW and JPEG. In fact, the image data consisting of the above four bands were acquired twice by UAS imaging. S110 RGB acquired the true-color image data in single UAS imaging; another UAS imaging with the S110 NIR acquired the false-color image data that consists of the red $(570-690 \mathrm{~nm})$, green $(510-660 \mathrm{~nm})$ and NIR $(780-1000 \mathrm{~nm})$ bands and is therefore able to capture the amount of NIR radiation a surface reflects (Table 2). This is especially useful to calculate indices like the NDVI, SAVI, MCARI as reported by Joseph (2005).

Table 2 Flight information using eBee Ag (fixed wing) UAVs on April 26, 2018 at Kotmomin, Sargodha.

\begin{tabular}{|c|c|c|c|c|c|c|c|c|}
\hline \multirow{2}{*}{ Camera } & \multirow{2}{*}{ Platform (UAS) } & \multirow{2}{*}{ Flight speed $/ \mathrm{m} \cdot \mathrm{s}^{-1}$} & \multirow{2}{*}{ Altitude/m } & \multicolumn{2}{|c|}{ Percent overlap } & \multirow{2}{*}{ No. of images } & \multirow{2}{*}{ Image format } & \multirow{2}{*}{ Spatial resolution/cm } \\
\hline & & & & Side $/ \%$ & Forward/\% & & & \\
\hline MultiSpec 4C & eBee Ag & 8 & 41 & 75 & 75 & 22 & 8 bit TIFF & 5 \\
\hline
\end{tabular}

To avoid geometric distortion due to low altitude, 22 overlapping pictures from each camera and fly were used for mosaicking to produce an ortho-image. The $75 \%$ frontal overlap and $75 \%$ side overlap were used as suggested by Gómez-Candón, De Castro, and López- Granados (2014). The flight plans were performed on the eMotion ${ }^{\circledR}$ software.In order to orient and relate UAS imagery to the ground, 46 ground control points (GCPs) were distributed across the kinnow field to obtain photogrammetric imagery with uniform horizontal and vertical accuracy. The GCPs were $25 \mathrm{~cm} \times 25 \mathrm{~cm}$ square, with a specific albedo for camera calibration, mounted on a $50-\mathrm{cm}$ post.

\subsection{UAVs data processing}

For each flight, images were georeferenced and elaborated using the Pix4D manager tool of the eMotion software. The eBee's supplied software to build a project using the drone's geotagged images. To create an accurately georeferenced ortho-mosaicked image of the kinnow field, the multiple overlapped images were stitched together and ortho-rectified. In the laboratory, data processing (ortho-mosaicking) of acquired images was performed with Pix4D software package, to generate ortho-images. Pix4D incorporates scale-invariant feature transform algorithm to match key points across multiple images (Küng et al., 2011; Lowe, 2004) and processes data in three key steps: (1) initial processing (camera internals and externals, automated aerial triangulation, bundle block adjustment); (2) point cloud densification; and (3) (digital surface map [DSM]) and ortho-mosaic generation. The exterior position and orientation parameters of the UAS, referring to the roll, pitch and yaw angles of every overlapped image, were provided by the UAS inertial system. These parameters were used as input data to the Pix4D software for ortho-rectification by aero-triangulation and mosaicking. Aero-triangulation involves the transformation of image coordinates to ground coordinates through a set of GCPs that are clearly visible in the set of images. This step consists of forcing an exact match between image and GCPs coordinates implemented in the software. Additional auto tie points were generated automatically to improve the aero-triangulation results. Ortho-images and DSMs were produced from the flights; DSMs were interpolated from the densified point clouds and used to ortho-rectify the individual images. The final step combined the ortho-rectified images to form a seamless ortho-image mosaic.
The ortho-mosaic was georeferenced to UTM-WGS84 zone 43N Pakistan. The final outputs were an RGB (visible) GeoTIFF with a resolution of $3.5 \mathrm{~cm} /$ pixel (Figure 3 ) then the masked the only the kinnow area for calculating vegetation indices (Figure 4). The NDVI, TNDVI, SAVI, MSAVI2 and MCARI2 layers were generated in raster calculator from extracted red (R) and NIR channels. The index calculator function of Pix4D was used for generating VIs maps (Figure 5). To optimize internal camera parameters, such as focal length, principal points, lens distortions, a calibration file (certified by SensFly on canon S110 NIR camera) was uploaded in the software.

The 46 GCPs with a known albedo for Red, Green and NIR channel (reflectance panel) were used to calibrate the camera to achieve uniform quality of image (exposure and brightness) and for atmospheric correction in the software section processing options, point 3 DSM, Ortho-mosaic, Index and for creating VIs map. The resolution of reflectance map (NDVI, TNDVI, SAVI, MSAVI2 and MCARI2) has been set at $3.5 \mathrm{~cm} /$ pixel GeoTIFF. GeoTIFF images and georeferenced sampling data were processed for agronomic purpose with ERDAS 14.0

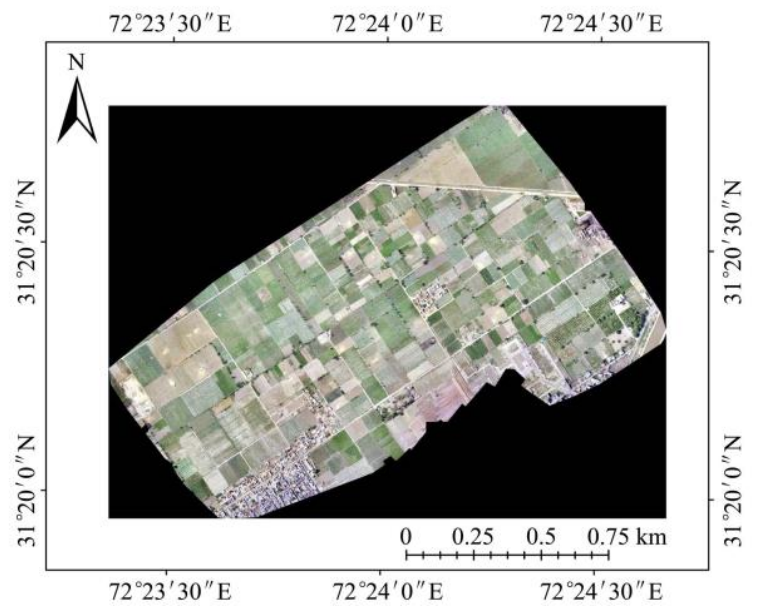

Figure 3 Ortho-mosaic image of the study area 


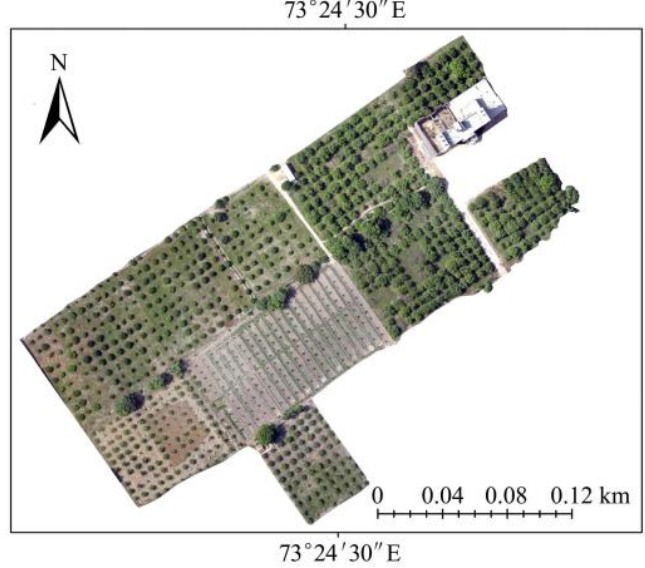

Figure 4 Masking the the kinnow orchard from ortho-mosaic image

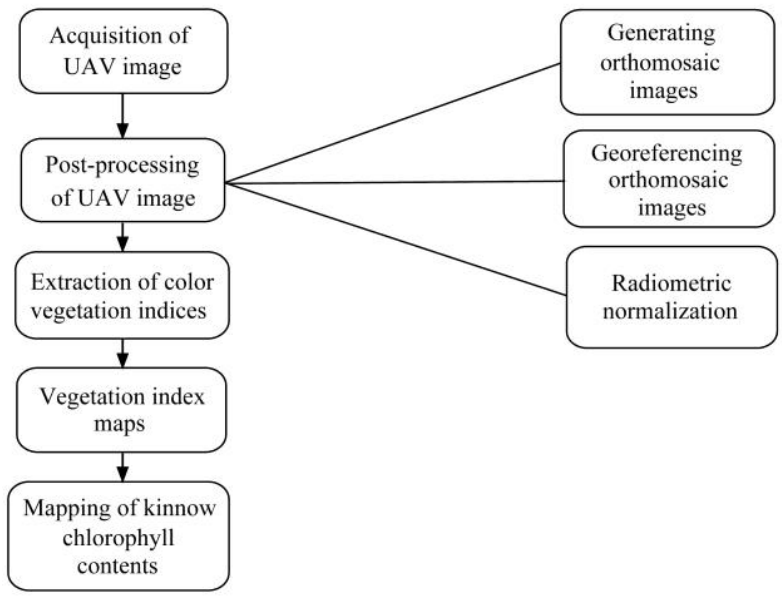

Figure 5 Schematic diagram of the study

\subsection{Spectral Vegetation Indices}

NDVI was originally developed to provide information about vegetation, (biomass and LAI) and chlorophyll content in leaves (Rouse et al., 1974). Till now it has been used in a variety of applications including change detection, crops prediction, yield estimation, and most importantly disease monitoring by several scientists (Bulanon et al., 2013; Grieve et al., 2015; Ramsey et al., 1995; Wade et al., 1994). The index is sensitive to the presence of green vegetation and can be defined by equation (1).

$$
\mathrm{NDVI}=\frac{\mathrm{NIR}-\mathrm{Red}}{\mathrm{NIR}+\operatorname{Red}}
$$

\subsection{Transformed Normalized Difference Vegetation Index} (TNDVI)

Transformed Normalized difference vegetation index (TNDVI) was proposed by tucker, 1979. This index is robust for biomass and vegetation.

$$
\mathrm{TNDVI}=\mathrm{SQRT}\left(\frac{\mathrm{NIR}-\operatorname{Red}}{\mathrm{NIR}+\operatorname{Red}}+0.5\right)
$$

\subsection{Soil adjusted vegetation index (SAVI)}

SAVI was proposed by Huete (1988) to account for the optical soil properties in the plant canopy reflectance. The SAVI was calculated according to Equation (3).

$$
\mathrm{SAVI}=\left[\frac{\mathrm{NIR}-\operatorname{Red}}{\mathrm{NIR}+\operatorname{Red}+L}\right] \times(1+L)
$$

where, $L$ is a constant. SAVI involves a constant $L$. The constant $L$ was introduced in order to minimize soil brightness. SAVI defined the soil-adjustment factor $L$ in the SAVI equation varying from 0 to 1 according to the canopy density. $L$ decreases with increases in vegetation amount. For $L=0$, SAVI is equal to NDVI. According to above cited papers, we have set the $L$ value at 0.5 for this study.

\subsection{Modified Soil Adjusted Vegetation Index (MSAVI2)}

MSAVI2 was introduced by Qi. et al. (1994) to minimize soil-induced variations in vegetation indices and can be expressed by following equation (4).

MSAVI2 $=(2 \times N I R+1-S Q R T((2 \times N I R+1) 2-8 \times(N I R-R E D)))(4)$

\subsection{Modified Chlorophyll Absorbed Ratio Index}

MCARI is the modified form of CARI. To enhance the ability of CARI that converts into MCARI. Modified chlorophyll absorbed ratio was obtained by the following equation as described by (Daughtry et al., 2000).

MCARI $=[(\mathrm{R} 700-\mathrm{R} 670)-0.2 *(\mathrm{R} 700-\mathrm{R} 550) *(\mathrm{R} 700 / \mathrm{R} 670)]$

\subsection{Statistical Analysis and Mapping}

Different vegetation indices were calculated by using the mean values of the reflectance in green, red and NIR portion of the electromagnetic spectrum of UAVs. The derived vegetation indices; NDVI, TNDVI, SAVI, MSAVI2 and MCARI2 proposed different band ratios which demonstrated the feasibility of estimating the kinnow chlorophyll contents. Statistical analysis was performed to assess and established relationship between UAVs derived parameter and ground-truthing chlorophyll by performing regressional model. Probability and spatial distribution of chlorophyll content was mapped to identify the Coefficient of Determination $\left(R^{2}\right)$ between various vegetation indices and chlorophyll content. The correlation coefficient was used to identify the most sensitive vegetation indices to chlorophyll then the highest $R^{2}$ values were used to develop regression equations to predict chlorophyll content from spectral reflectance data.

\section{Results and Discussion}

\subsection{NDVI and kinnow tree leaves chlorophyll content}

Normalized difference vegetation index (NDVI) is that in which we caluclate the photosynthtical absored radation. The NDVI map showed the status of the kinnow orchard at the komomin, district Sarghoda. NDVI map showed the minimum value of 0 which ranged to highest value of +0.9923 . Water bodies and builtup area has negative values where as strong postive values show high dense green vegatative area (Figure 6). In pothwar region district Chakwal found impontant value about agriculture aspect so higher values of NDVI in map showed the rich vegatation.

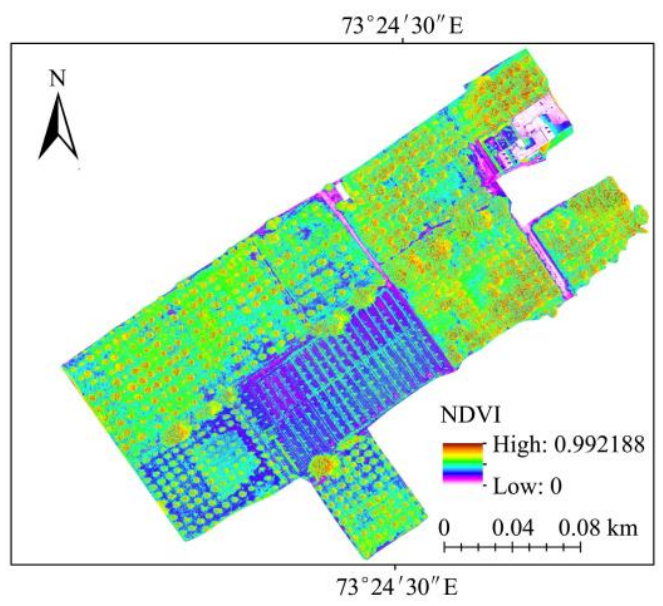

Figure 6 NDVI map of the kinnow orchard

A positive and linear relationship was observed between NDVI and the kinnow tree chlorophyll contents. The regression 
accounted for $79 \%$ of the variation in the data $\left(R^{2}=0.79\right)$ which means that the NDVI value varies with leaf chlorophyll contents (Figure 7). The regressional model $(Y=188.93 X)$ interprets the significant interaction between NDVI and the the kinnow tree leaves chlorophyll contents. The results are also supported by (Hashmi et al., 2011) with $R^{2}$ values of $86 \%$.

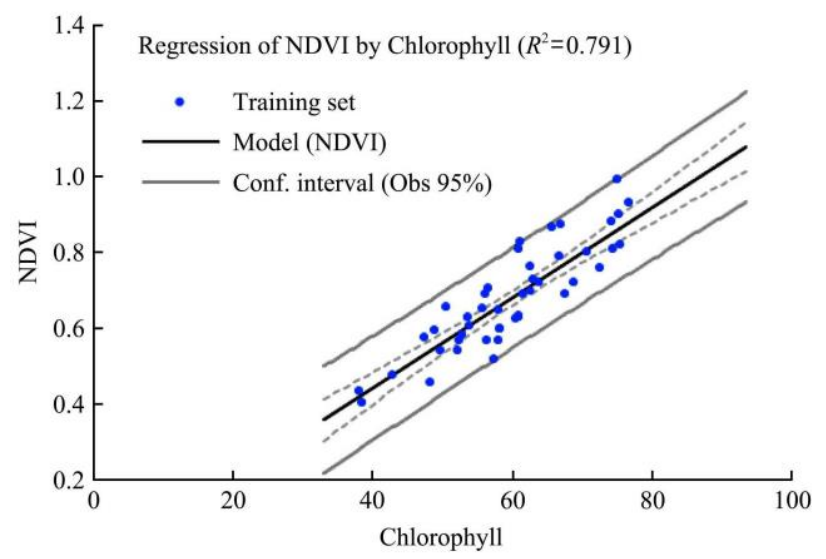

Figure 7 Relationship between NDVI and chlorophyll content of the kinnow tree leaves

\subsection{TNDVI and kinnow tree leaves chlorophyll content}

Transformed normalized difference vegetation index is used for the measurement of greenness and biomass in the crops. The TNDVI map showed variation across the kinnow orchard in kotmomin at district Sargodha. Minmum value was 0.707 and maximum value was 1.2247 (Figure 8). The kinnow trees showewd higher content of greenexx and NDVI is usally satuare under high greeness content. The TNDVI showed better range for estimation of greeness indexs as compared to NDVI.

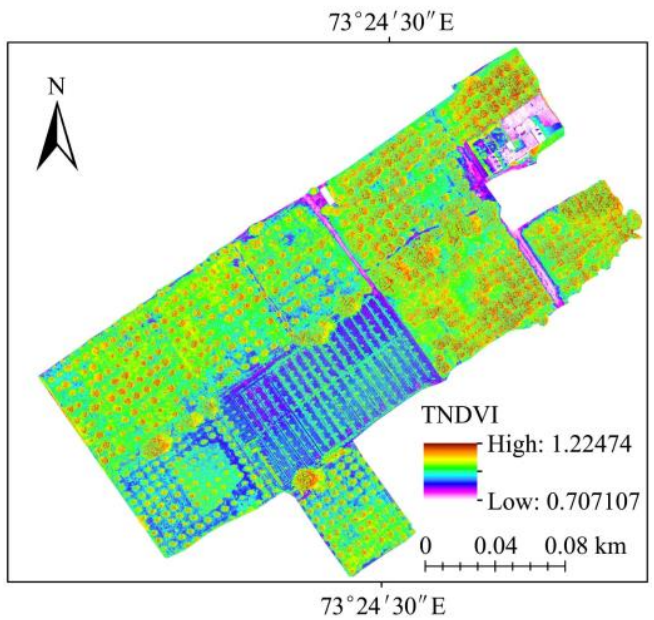

Figure 8 TNDVI map of the kinnow orchard

The results of regression model interpreted the significant relationship between TNDVI and the kinnow tree leaves chlorophyll contents. The regression model $(Y=225.44 X)$ accounted for $85 \%$ of the variation in the data $\left(R^{2}=0.85\right)$ which means that the TNDVI values varied with leaf chlorophyll contents. The value of $R^{2}=0.85$ showed the accuracy between the chlorophyll content and TNDVI which explained the fitness of model (Figure 9). The chlorophyll concentration varied with plant age, soil available nutrients and many other factors. The results are also supported by the relationship between chlorophyll content and TNDVI (Blackburn \& Steele, 1999; Bell et al., 2004; Li-Hong et al., 2007) with $R^{2}$ value of $0.72,0.88$ and 0.82 respectively.

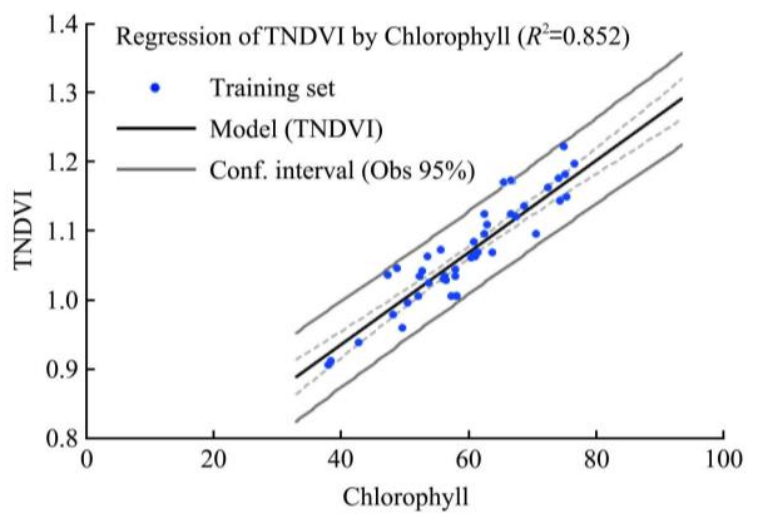

Figure 9 Relationship between TNDVI and chlorophyll content of the kinnow tree leaves

\subsection{Soil adjusted vegetation index (SAVI) and kinnow tree leaves chlorophyll content}

Map of SAVI index showed the variations across the kinnow orchard. In the map, Figure 10 showed that lower value was zero and higher value was 1.4971 . The results of regression model interpreted the significant relationship between SAVI and the kinnow tree leaves chlorophyll contents. SAVI also showed positive relationship with the the kinnow leaves tree chloropyll contents but was less as compared to NDVI and TNDVI. SAVI representd $R^{2}$ value of 0.73 with regression model (Figure 11). The value of $R^{2}=0.73$ showed the accuracy between the chlorophyll content and SAVI which explained the fitness of model (Figure 11).

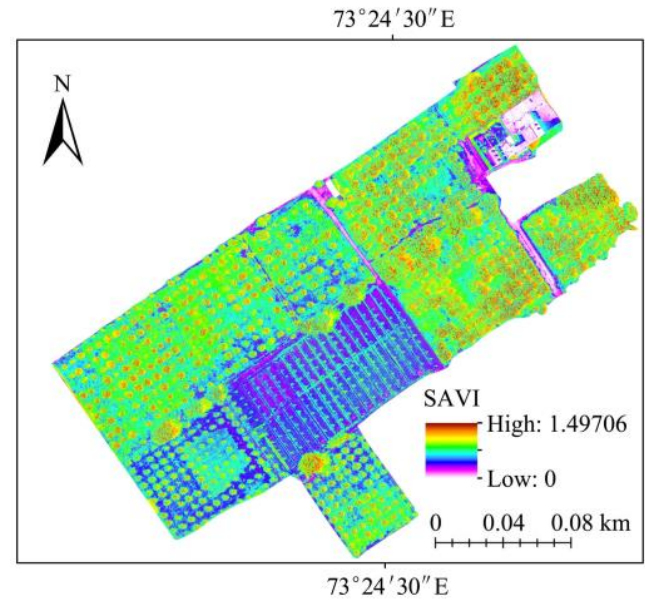

Figure 10 SAVI map of the kinnow orchard

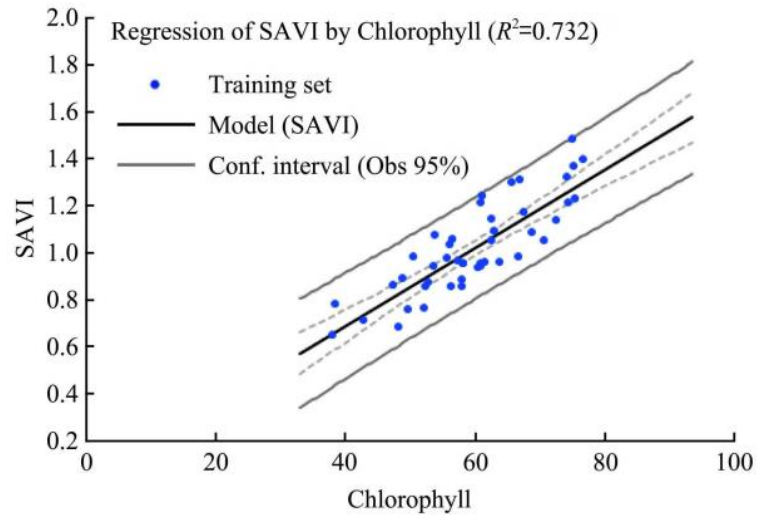

Figure 11 Relationship between SAVI and chlorophyll content of the kinnow tree leaves 
3.4 Modified Soil adjusted vegetation index (MSAVI2) and kinnow tree leaves chlorophyll content

MSAVI2 is modified form of SAVI. The map of MSAVI2 showed the chlorophyll variations across the kinnow orchard. MSAVI2 values ranged from 0 to 0.9961 which classified the study area from low to dense vegetation areas (Figure 12). Low values of chlorophyll contents usually responded with low vegetative or non-vegetative areas while higher value of chlorophyll content showed area with dense vegetation.

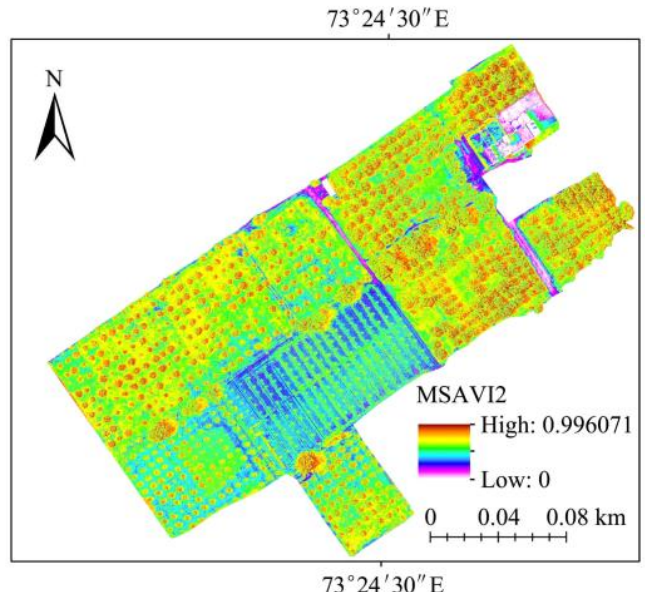

Figure 12 MSAVI2 map of the kinnow orchard

MSAVI2 values varied across the whole field of the kinnow orchard at kotmomin. A positive and strong relationship found between the kinnow chlorophyll content MSAVI2 with higher coefficient of determination $\left(R^{2}\right)$ value of 0.89 (Figure 13). This index proved more accuracy of estimation of chlorophyll content by reducing the background noise effectively. MSAVI2 removed the soil background noise and improved the prediction efficiency. MSAVI2 proved more robust index for estimation of the kinnow chlorophyll content among all other indices used in this study. The relationship between MSAVI2 and the kinnow chlorophyll content was found very strong as compared to TNDVI, NDVI, SAVI and MCARI2 The study results are also supported by (Haboudane et al., 2004; Zhang et al., 2014) with $\mathrm{R}^{2}$ of $55 \%$ and $66.74 \%$ respectively.

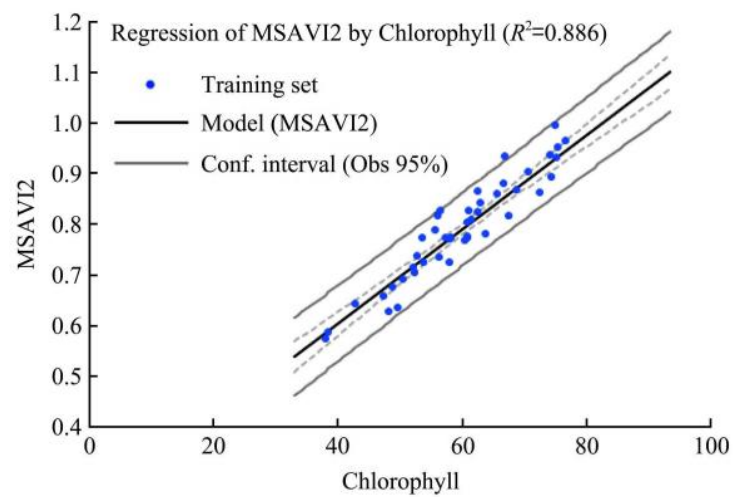

Figure 13 Relationship between MSAVI2 and chlorophyll content of the kinnow tree leaves

\subsection{Modified chlorophyll absorption ration index (MCARI2)} and kinnow tree leaves chlorophyll content

MCARI is the modified form of CARI. To enhance the ability of CARI that converts into MCARI2. MACRI2 values varied across the whole of field of the kinnow orchard (Figure 14).

MCAR2I values ranged from minimum value $0-0.04003$ to maximum value 1.2542. Low values of chlorophyll contents usually responded with low vegetative or non-vegetative areas while higher value of chlorophyll content showed area with dense vegetation (Figure 14).

$73^{\circ} 24^{\prime} 30^{\prime \prime} \mathrm{E}$

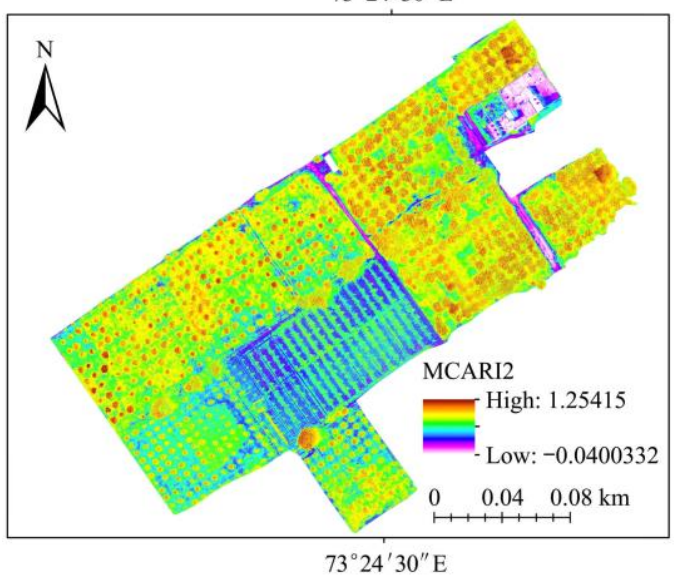

Figure 14 MCARI2 map of the kinnow orchard

There is positive and linear relationship between MCARI2 and the kinnow chlorophyll contents. The regression model accounted for $81 \%$ of the variation in the data $\left(R^{2}=0.81\right)$ which means that the MCARI2 values varies with leaf chlorophyll contents. The value of $R^{2}=0.81$ showed the accuracy between the chlorophyll content and MCARI2 which explained the fitness of model (Figure $15)$.

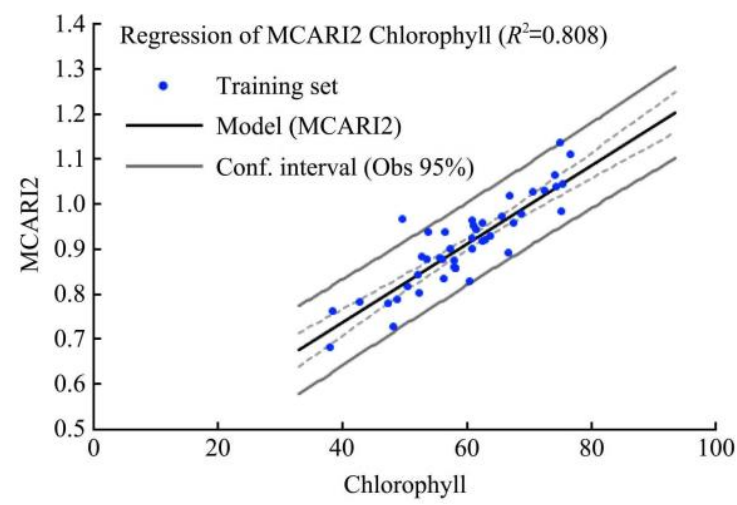

Figure 15 Relationship between MCARI2 and chlorophyll content of the kinnow tree leaves

All indices (NDVI, TNDVI, SAVI, MSAVI2 and MCARI2) showed positive and linear relationship with kinnow tree leaves chlorophyll contents while MSAVI2 showed strong relationship $\left(R^{2}=0.89\right)$ with regression model $Y=14.96 X$ (Figure 12). In this study, MSAVI2 showed improved results as compared to others studies (Liao et al., 2013).

\subsection{Probability Map of Chlorophyll Contents}

The probability Map of chlorophyll content showed the status of spatial distribution of chlorophyll contents in the kinnow orchard at Kotmomin. The probability map predicted the value of chlorophyll contents for future years. Threshold value play important role in the prediction of the value. The probability map value ranged from 0 to 1 . When the values lies near to 1 , represented higher chlorophyll content. In the legend showed that more values were lie near to 1 , which explained higher value of chlorophyll content shown in map with Red color. Some area shown in Blue color in the map, explained the lower values for chlorophyll contents. This predicts the vegetation pattern for the whole field of the kinnow orchard in district Sargodha (Figure 16). 
$73^{\circ} 24^{\prime} 30^{\prime \prime} \mathrm{E}$

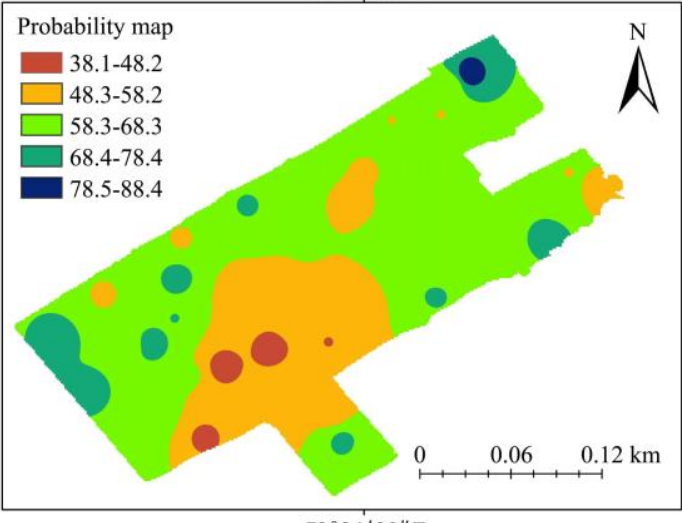

$73^{\circ} 24^{\prime} 30^{\prime \prime} \mathrm{E}$

CDROM. Retrieved March 12, 2012 from http://www.isprs.org/ proceedings/XXXVI/5-W1/papers/11.pdf.

[4] Gitelson, A. A., A. Vina, V. Ciganda, D. C. Rundquist and T. J. Arkebauer. Remote estimation of canopy chlorophyll content in crops. Geophysical Research Letters, 2005; 32(8).

[5] Gitelson, A. A., Y. Gritz and M. N. Merzlyak. Relationships between leaf chlorophyll content and spectral reflectance and algorithms for non-destructive chlorophyll assessment in higher plant leaves. Journal of plant physiology, 2003; 160(3): 271-282.

[6] Hu, B., S. E. Qian, D. Haboudane, J. R. Miller, A. B. Hollinger, N Tremblay and E. Pattey. Retrieval of crop chlorophyll content and leaf area index from decompressed hyperspectral data: The effects of data compression. Remote Sensing of Environment, 2004; 92(2): 139-152.

[7] Huang Y., Hoffmann, W. C., Lan, Y., Wu, W., Fritz, B. K.. Development of a spray system for an unmanned aerial vehicle platform. Appl. Eng. in Ag., 2009; 25, 803-809.

[8] Hunt, E. R., Cavigelli, M., Daughtry, C. S. T., McMurtrey, J. E., \& Walthall, C. L. Evaluation of digital photography from model aircraft for remote sensing of crop biomass and nitrogen status. Precision Agriculture, 2005; 6, 359-378.

[9] Jensen, T., A. Apan, F. R. Young, L. Zeller, and K. Cleminson. Assessing grain crop attributes using digital imagery acquired from a low-altitude remote controlled aircraft. In Proc. Spatial Sci. 2003 Conf. Canberra, Australia: Spatial Sciences Institute, Deakin ACT, Australia.

[10] Johnson, L. F., Herwitz, S. R., Lobitz, B. M., \& Dunagan, S. E. Feasibility of monitoring coffee field ripeness with airborne multispectral imagery. Applied Engineering in Agriculture, 2004; 20, 845-849.

[11] L. Hassan-Esfahani, A. Torres-Rua, A. M. Ticlavilca, A. Jensen, M McKee, "Topsoil Moisture Estimation for Precision Agriculture Using Unmanned Aerial Vehicle Multispectral Imagery", 2014 IEEE International Geoscience and Remote Sensing Symposium, 2014.

[12] Laliberte, A. S., \& Rango, A. Image processing and classification procedures for analysis of subdecimeter imagery acquired with an unmanned aircraft over arid rangelands. GIScience \& Remote Sensing,

chlorophyll contents taken by chlorophyll meter (SPAD-502 Minolta). Linear regression analyses were developed between different vegetation indices and the kinnow tree leaves chlorophyll contents to develop prediction model for kinnow tree leaves chlorophyll content.. MSAVI2 and TNDVI showed strong and positive relationship with the kinnow tree leaves chlorophyll content with value of $R^{2}=0.89$ and 0.85 respectively among all other indices (MCARI2, NDVI, SAVI) used in the study. MSAVI2 proved to be more robust index for accurately estimation of kinnow tree leaves chlorophyll content in real time. The results showed the efficiency of multispectral UAVs for mapping spatial differences in chlorophyll content at the regional scale.

\section{Acknowledgment}

This research was funded by the Science and Technology Plan Project of Guangzhou city, China, grant number 201807010039, and the Science and Technology Plan Project of Guangdong province, China, grant number 2018A050506073. This study is joint effort between National Center for International Collaboration on Precision Agricultural Aviation Pesticides Spraying Technology, South China Agriculture University, Guangzhou, China and Department of Agronomy, PMAS-Arid Agriculture University Rawalpindi, Pakistan. The author is indebted to all the students who participated in the field data collection campaign.

\section{[References]}

[1] Barton, C. V. A theoretical analysis of the influence of heterogeneity in chlorophyll distribution on leaf reflectance. Tree Physiology, 2001; 21(12-13): 789-795.

[2] Blyenburgh, P. V. UAVs: An overview. Air \& Space Eur. 1999; 1(5): 43-47.

[3] Eisenbeiss, H. A mini unmanned aerial vehicle (UAV): system over and image acquisition. In: A. Gruen, Sh. Murai, T. Fuse, F. Remondino (Eds.). Proceedings of International Workshop on Processing and Visualization Using High-Resolution Imagery, XXXVI(5/W1), Pitsanulok, Thailand. 


\section{$2011 ; 48,4-23$}

[13] Le Maire, G., C. Francois and E. Dufrene. Towards universal broad leaf chlorophyll indices using prospect simulated database and hyperspectral reflectance measurements. Remote sensing of environment, 2004; 89(1): $1-28$.

[14] Moran, J. A., A. K. Mitchell, G. Goodmanson and K.A. Stockburger. Differentiation among effects of nitrogen fertilization treatments on conifer seedlings by foliar reflectance: A comparison of methods. Tree physiology, 2000; 20(16): 1113-1120.

[15] Richardson, A. D., S. P. Duigan and G. P. Berlyn. An evaluation of noninvasive methods to estimate foliar chlorophyll content. New phytologist, 2002; 153(1): 185-194.

[16] Rouse, J., R. Haas, J. Schell, D. Deering and J. Harlan. Monitoring the vernal advancement of retrogradation of natural vegetation, nasa/gsfc, type iii, final report. Greenbelt, 1974; 371.

[17] Sims, D. A. and J. A. Gamon. Relationships between leaf pigment content and spectral reflectance across a wide range of species, leaf structures and developmental stages. Remote sensing of environment, 2002; 81(2-3): 337-354.

[18] Swain, K. C., Thomson, S. J., \& Jayasuriya, H. P. W. Adoption of an unmanned helicopter for low altitude remote sensing to estimate yield and total biomass of a rice crop. Transactions of the ASABE, 2010; 53, 21-27.

[19] Tahir, M. N., J. Li, B. Liu, G. Zhao, Y. Fuqi and C. Chengfeng. Hyperspectral estimation model for nitrogen contents of summer corn leaves under rainfed conditions. Pak. J. Bot, 2013; 45(5): 1623-1630.

[20] Wu, J. D., Wang, D., \& Rosen, C. J. Comparison of petiole nitrate concentrations, SPAD chlorophyll readings, and QuickBird satellite imagery in detecting nitrogen status of potato canopies. Field Crops Research, 2007b; 101, 96-103.

[21] Yuan, W., S. Liu, G. Zhou, G. Zhou, L.L. Tieszen, D. Baldocchi, C. Bernhofer, H. Gholz, A. H. Goldstein and M. L. Goulden. Deriving a light use efficiency model from eddy covariance flux data for predicting daily gross primary production across biomes. Agricultural and Forest Meteorology, 2007; 143(3-4): 189-207. 